\title{
Environnement et développement : approches scientifiques, structuration du domaine et coévolution des recherches
}

\author{
Alain Pavé \\ Les recherches sur l'environnement portent sur des questions très diverses et \\ mobilisent un large éventail de disciplines. Nombreux sont les organismes de \\ recherches, en France, qui y consacrent peu ou prou une partie de leur activité. \\ Ce foisonnement et cette apparente dispersion n'excluent pas une cohérence \\ d'ensemble d'une démarche qui, en fin de compte, conduit à lier de plus en plus \\ étroitement recherches concernant l'environnement et recherches \\ concernant le développement. Faire apparaître l'unité de ce champ de recherche \\ le rend plus intelligible et met en évidence les synergies à développer.
}

\section{$\overline{\text { ALAIN PAVÉ }}$ Professeur des Universités, directeur du programme interdisciplinaire de recherche \\ - Environnement, vie et sociétés - du CNRS, président du conseil scientifique de l'Orstom (Institut français de recherche scientifique pour \\ le développement en coopération) et président du conseil scientifique du Cirad (Centre de coopération internationale en recherche agronomique pour le développement)}

\footnotetext{
1 Préservation des ressources et maintien de la viabilité de l'environnement sont d'ailleurs, presque sûrement, des conditions

d'un développement s'étalant sur une - longue durée - ; mais il vaut mieux
} le préciser.

\section{Introduction}

Les approches scientifiques de l'environnement et du développement proviennent essentiellement de la prise de conscience de deux ensembles de questions : - Celui des atteintes à l'environnement des sociétés humaines liées au développement de ces sociétés (pollutions, perturbation des écosystèmes, urbanisation, effets sur la santé, renouvelabilité des ressources naturelles, etc.). Bien que cela paraisse quelquefois aller de soi, il est bon de répéter que les sociétés humaines assurent leur développement en prélevant des ressources dans leur environnement et, très généralement, en interagissant avec lui, tout en remarquant bien que ces interactions ne sont pas toujours négatives.

- Celui du développement des sociétés humaines, notamment des pays du sud (économies fragiles, hétérogénéité des modes de développement, solutions techniques et économiques mal adaptées, problèmes de nutrition, de santé et d'éducation, émergence de tensions politiques et conflits, etc.), mais aussi des interrogations sur les modes de développement des pays du Nord (transports, industries et agricultures gros consommateurs d'énergie et dispensateurs de pollutions, modes d'aménagement de l'espace, déréglementation, etc.).

Pendant longtemps, les problèmes, qu'ils soient de développement et surtout d'environnement, ont été abordés dans l'urgence et dans le court terme, sans évaluer les incidences des solutions choisies et en gardant plus on moins consciemment l'espoir que les sciences et les techniques, toutes puissantes, arriveraient bien au bout du compte à résoudre les nouveaux problèmes posés ou bien que la nature s'en chargerait. Par exemple, on ne peut nier les progrès spectaculaires de l'agriculture productiviste et son efficacité dans la réponse aux questions de la suffisance alimentaire ; en revanche, on n'a pas évalué à sa juste mesure les impacts environnementaux (pollutions diffuses par les engrais ou les pesticides, modifications de l'utilisation des terres et leurs effets, etc.). On n'en a pas, non plus, estimé et anticipé les conséquences socioéconomiques. Fuite en avant, laissant aux autres, en l'occurrence à la nature * bienfaisante ", aux voisins ou aux générations futures, le soin de résoudre ces nouveaux problèmes. Les questions sont particulièrement préoccupantes dans les pays du Sud.

La prise de conscience des risques encourus a conduit à de nombreuses initiatives. À l'échelon international, celles-ci ont été à l'origine de l'interpellation des mondes politiques, économiques, techniques et scientifiques. Citons pour mémoire le rapport du Club de Rome (1972, sur des travaux lancés dès les années 1960) ; le rapport Brundtland (1985) et l'émergence du concept de développement durable ; la Conférence des Nations unies sur l'environnement et le développement (CNUED : Stockholm, 1972, et Rio, 1992 : conventions sur l'effet de serre, sur les forêts et sur la biodiversité, rédaction de l'^Agenda 21 ", présentant les conditions, identifiées à l'époque, d'un développement durable) ; la Conférence sur la démographie (Le Caire, 1994) ; la Conférence d'Istanbul sur la ville (1996).

Aujourd'hui, avec l'accélération des changements, la question est : comment continuer à assurer un développement aux sociétés de notre planète, pour nous et les générations futures, qui prenne nécessairement en compte la pérennité ou l'extension des ressources et la viabilité de notre environnement ?'

Ce texte est issu d'une réflexion menée à l'occasion du colloque international - Écologie, sociologie, économie * qui s'est tenu en mai 1996 à Saint-Quentin-en-Yvelines. Les organisateurs de ce colloque, notamment Sylvie Faucheux, m'avaient demandé d'intervenir sur le thème - Coévolution entre les recherches sur l'environnement et les recherches sur le développement. - J'étais depuis longtemps convaincu que ces champs de recherche devaient se rapprocher, ce fut l'occasion d'approfondir le point de vue qui est présenté ici. 
De cette interrogation est venue l'idée de développement durable.

À noter que ces initiatives couplent explicitement les problèmes d'environnement et ceux de développement. Pendant une longue période, ce n'était pas toujours le cas pour la recherche scientifique malgré les nombreuses déclarations d'intention ${ }^{2}$. Aujourd'hui, ce lien est largement admis et explicité par la communauté scientifique (voir Environnement et développement). II éclaire, de façon plus générale, les relations entre les sociétés humaines et leur environnement. Néanmoins, ce lien se caractérise par ses - non linéarités ${ }^{3}$, ce qui complique la compréhension globale du couplage entre les dynamiques sociales et les dynamiques environnementales, entre les sociétés et leur environnement. Enfin et comme nous allons le voir, l'approche scientifique de ces problèmes met en œuvre des ensembles différents de disciplines. Au-delà des discours et des intentions, nous retrouvons encore les difficultés du travail interdisciplinaire, de sa nécessité, de sa pertinence et de sa mise en pratique.

\section{Structuration progressive de la recherche scientifique}

En pratique, les recherches concernant l'environnement et celles centrées sur le développement procèdent classiquement de trois points de vue distincts : celui des sciences de la nature, celui des sciences de I'homme et de la société, celui des sciences de l'ingénieur (cf. encadré). Par ailleurs, on peut distinguer les recherches sur l'environnement, les recherches pour l'environnement, les recherches sur le développement et les recherches pour le développement :

- Les recherches sur l'environnement visent à identifier et à comprendre les mécanismes naturels et anthropiques qui président à l'évolution de cet environnement, à évaluer leurs rôles réciproques et à esquisser cette évolution.

- Les recherches pour l'environnement sont de nature plus techniques et technologiques et visent à prévenir les effets négatifs de l'activité humaine sur l'environnement (énergies nouvelles, technologies propres, ingénierie des milieux naturels, techniques d'aménagement, etc.).

- Les recherches sur le développement ont pour objectif de cerner les processus qui président à l'évolu- tion économique, technologique, culturelle, sanitaire, etc. des sociétés humaines.

- Les recherches pour le développement s'intéressent également aux solutions techniques, socioéconomiques et réglementaires qui autorisent et facilitent le développement des sociétés humaines, dans leurs diversités sociales et environnementales.

Recherches sur l'environnement (plutôt en amont) et recherches pour l'environnement (plutôt en aval) doivent être en forte interaction, de même, les recherches sur le développement et les recherches pour le développement.

Ces interactions impliquent déjà un premier niveau d'interdisciplinarité, qu'on a retrouvé et qu'on retrouve par exemple dans les programmes de recherche sur l'environnement, comme ceux du CNRS. La prise en compte simultanée des questions de développement et des problèmes d'environnement conduit à un deuxième niveau d'interdisciplinarité. Classiquement et très schématiquement, ces recherches sont organisées de la façon suivante :

- Les recherches sur l'environnement et celles intégrant fortement des préoccupations environnementales sont principalement menées dans le cadre des sciences de la nature (approches * géophysicochimiques et bioécologiques - des sciences de la planète et des sciences de la vie). Elles sont assez bien structurées sous forme de programmes nationaux et internationaux (cf. encadré). De très nombreux pays participent à cet effort collectif et notons que presque tous les organismes de recherche français revendiquent des recherches sur l'environnement.

- Les recherches pour l'environnement tentent de trouver des solutions techniques ou réglementaires aux problèmes d'environnement. Elles relèvent des sciences de l'ingénieur au sens large (incluant aspects industriels, techniques agricoles, aménagement et gestion des milieux). Cependant, certains secteurs des sciences de l'homme et de la société sont aussi impliqués : disciplines sociologiques et juridiques (sociétés, normes et règles), économie, géographie, etc. ${ }^{4}$. En France des instituts de recherches affichent très clairement ces préoccupations. Par exemple : le Cemagref (Centre d'étude du machinisme agricole, du génie rural, des eaux et des forêts) ou l'Inra (Institut national de la recherche agronomique). Le programme Ecotech du CNRS s'intéresse aux technologie industrielles, à l'énergie et aux transports.

- Les recherches sur le développement font essentiellement appel aux sciences de l'homme et de la

\section{Environnement et développement}

Lors du récent colloque international : * Quel environnement au xxl $1^{\mathrm{e}}$ siècle ? Environnement, long terme et démocratie (Fontevraud, France, septembre 1996), il est apparu clairement que la nécessité d'expliciter le lien avec le développement lors des études sur l'environnement est maintenant très largement admise (et, symétriquement, pour celui avec l'environnement lors des études sur le développement). Ce lien est valable du local au global. Ainsi, d'une part, les travaux des économistes sur les modifications de l'effet de serre et les risques climatiques et, d'autre part, la prise en compte des aspects socioéconomiques par les physiciens des changements globaux illustrent cette dualité environnement-développement. On pourra se référer à :

Hourcade J.-C. (1994). * Analyse économique et gestion des risques climatiques * Natures, Sciences, Sociétés 3, 2, 202-211

Godard O. (1993). - Quel régime international pour les émissions de gaz à effet de serre - Natures, Sciences, Sociétés1, 1, 25-33

Fontan J. (1994). - Changements globaux et développement *. Natures, Sciences, Sociétés 2, 2, 153-155

${ }^{2}$ On retiendra néanmoins que la notion d'écodéveloppement précéda celle de développement durable. Les efforts faits dans cette direction ont surtout concerné les sciences sociales malgré l'intitulé impliquant clairement lécologie, science du vivant. À ce sujet on pourra consulter l'article : - Environnement développement, marché. Pour une économie anthropologique entretien avec Ignacy Sachs - par Jacques Weber (1994) in Natures Sciences Sociétés 2, 3, 258-265.

${ }^{3}$ Ce terme quelque peu ésotérique, ayant un sens précis en mathématiques. est de plus en plus employé pour dire, très schématiquement, qu'il n'y a pas proportionnalité des effets par rapport aux causes et que la relation entre causes et effets est plus complexe. Les dynamiques des systèmes - non linéaires - revêtent aussi une apparence complexe (par exemple, en se traduisant par des trajectoires irrégulières).

${ }^{4}$ Les recherches pour et sur l'environnement posent des problèmes méthodologiques, d'organisation du travail e de formation scientifique et technique. Ces questions ont été abordées dans Pavé A., Jollivet M. (1993). - About Nature science and society: the example of the environment. A new deal for scientists and engineers. Proceedings of the International Symposium: The Culture of Engineering in a Rapidly Changing World, University of Californi,a Berkeley, Conf. Grandes Écoles et MIT. U.C. Berkeley, 168180. 
Figure 1. Environnement et développement sont en ètroite interaction. Dans le contexte présent, ces deux termes sont relatifs à Thomme, aux sociètés humaines environnement de Ihomme, développement des sociétés humaines. Cette notion đ'environnement est valable de l'échelle locale à léchelle planètaire : lenvironnement local est relatif a lindividu ou au petit groupe dindividus (par exemple, une communauté villageoise) : lenvironnement global (ou planétaire) est relatif a lensemble des sociétés humaines de la planète Pour le développement, l'effet d'échelle joue également. On parle, par exemple, de développement local, régional ou planétaire. Dans les deux cas Ihétérogénéité ide

renvironnement des modes et conditions du développement) est source de problemes, mais aussi de richesses si on admet de les prendre en compte et de ne pas rechercher systematiquement des processus duniformisation ou dhomogene. sation. Comme exemples đhétérogènéité on citera lhétêrogènéité spatiale des systèmes naturels et sociaux. Thétérochronie ou hétérogénéité temporelle des processus, Thẻtérogénéité des espèces des systèmes écologiques, Thétérogénéite culturelle des systèmes sociaux, etc.

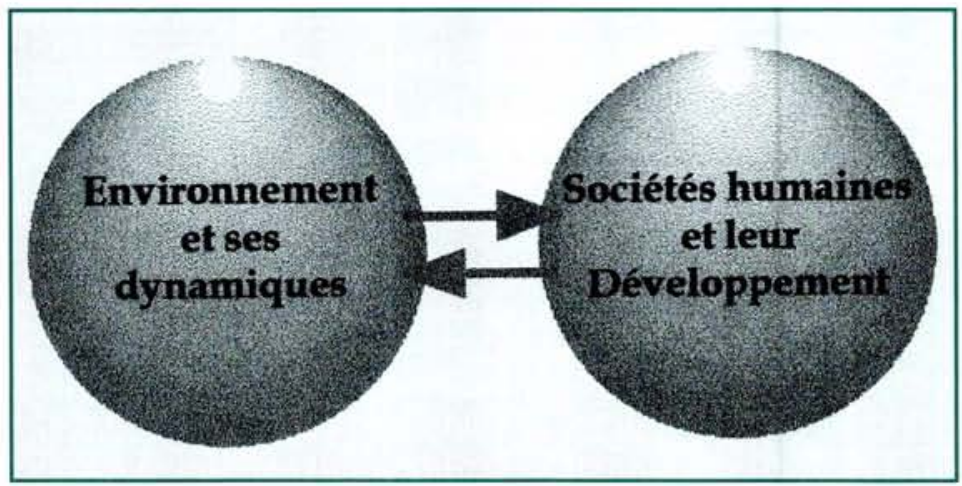

société, en particulier à l'économie, mais pas exclusivement ${ }^{5}$

- Les recherches pour le développement, comme pour l'environnement, sont principalement menées dans le cadre des sciences de l'ingénieur.

Recherches sur le développement et recherches pour le développement sont de plus en plus fréquemment confondues dans l'expression * recherches au service du développement ". Ces recherches sont partiellement structurées ${ }^{6}$. Ainsi, en France, deux organismes de recherche affichent explicitement des ambitions sur ce sujet pour les régions intertropicales (y compris les Dom-Tom), à savoir l'Orstom (Institut français de recherche scientifique pour le développement en coopération) et le Cirad (Centre de coopération internationale en recherche agronomique pour le développement). Au CNRS, Le Pir-EVS mène des travaux très largement interdisciplinaires qui couplent de plus en plus, mais pas exclusivement, les questions

${ }^{5}$ Par exemple, on peut aborder des questions relatives aux populations humaines avec un regard bioécologique. C'est ainsi qu'émerge l'- écologie humaine $\cdot$. On peut aussi aborder des problèmes de milieux très artificialisés, comme le milieu urbain. avec le même type d'entrèe, ce qui conduit à définir les contours d'une

- écologie urbaine Cf., par exemple : Legay J.M. (1991)

- Quelques hypothèses à débattre en écologie urbaine - Actes du colloque d'écologie urbaine, Mions (Rhône) France, 9-11.

${ }^{6}$ En France, le Comité national de coordination pour la recherche au service du développement fait le point sur ces sujets et contribue grandement à la réflexion à la fois sur le contenu de la recherche et sur sa mise en œuvre a travers le dispositif national de recherche publique. changement global - (un PIGB social) méritent d'être suivies et soutenues.

Les problèmes de développement sont souvent conditionnés par des aspects santé et réciproquement le niveau sanitaire d'une population est une des conditions de son développement. Pour les organismes de recherche français, I'Orstom a une action très pertinente dans ce domaine ; on retiendra aussi l'excellente contribution du réseau des Instituts Pasteur. L'OMS (Organisation mondiale de la santé) intervient au niveau international. De même pour l'éducation et la formation où les réseaux universitaires jouent un rôle important (par exemple, l'Aupelf/Uref), ainsi que des initiatives de certaines communautés scientifiques (par exemple, le Centre international de mathématiques pures et appliquées (Cimpa)).

\section{Recherches sur l'environnement et ses dynamiques, recherches sur les sociétés humaines et leur développement}

Sur des sujet aussi difficiles il est nécessaire d'expliciter les notions et les champs de recherche.

Figure 2. Environnement et développement : les différents aspects du problème. Les sociétés humaines qui veulent assurer leur développement concoivent et mettent en cuvre un ensemble de dispositifs techniques ou réglementaires (les outils ef moyens đu développement). Lutilisation de ces outils conduit à changer les états et les dynamiques sociales et environnementales. Lienjeu actuel est de concevoir des outils et des règles dont les applications soient - bénéfiques - aux sociétés humaines et à leur environnement dans le long terme et prenant en compte la diversité des situations sociales et environnementales. (1) et (2) sont les interactions qui ne mettent pas en cause directement les médiateurs techniques (aspects bio-écologiques de Thomme) ; (3) et (4) explicitent les relations entre sociétés humaines et leurs techniques (conception et effet économique et social, par exemple sut remploi) ; (5) et (6) representent dune part faction les effets directs ou indirects de Iutilisation ou de I application des techniques et des règlementations sur lenvironnement et, dautre part. linfluence environnementale sur les dispositifs techniques. par exemple. la dégradation - spontanée - de ces dispositifs. 


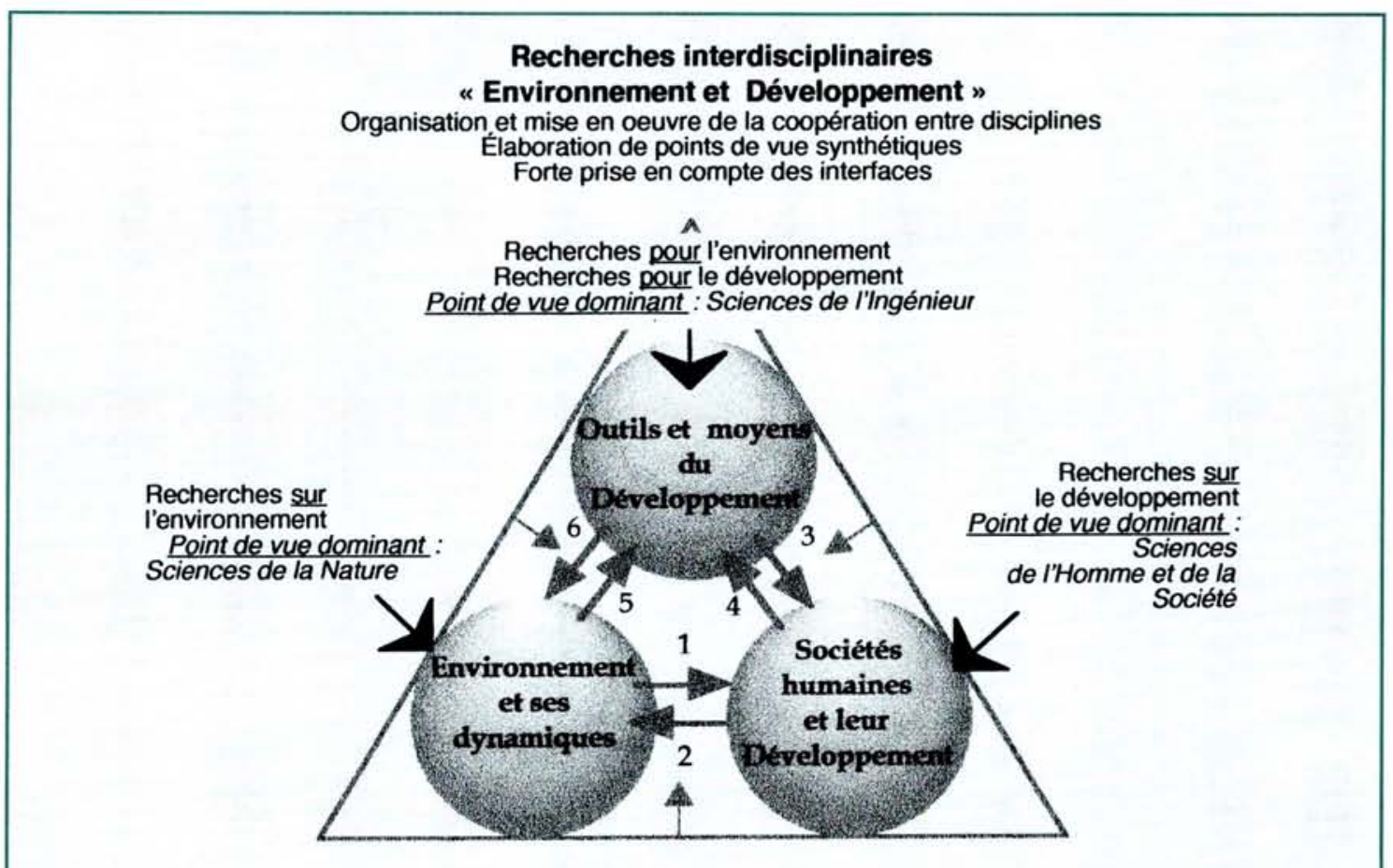

Figure 3. Organisation du travail scientifique Les questions denvironnement et de développement sont abordées par la recherche scientifique suivant trois points de vue distincts et complementaires : celui des sciences de la nature, celui des sciences de lhomme et de la sociéte celui des sciences de lingénieur. Classiquement. ces points de vue restent fortement centrès sur leurs objets et se croisent peu. Cependant lensemble · outils et moyens du dèveloppement - implique certes les sciences de lingénieur, mais intègre aussi des approches juridiques et régiementaires relevant des sciences de Thomme et de la socièté, ainsi que des aspects liés aux - sciences de la nature - (par exemple, Iagronomie, Ihydrologie, etc). Par ailleurs et ce n'est pas encore explicite dans ce type de schema. le recours de plus en plus fréquent a la modélisation (domaine qui traverse ces pratiques) implique de plus en plus les sciences mathèmatiques et linformatique. On sait aussi que lincomplètude des approches conduit à des résultats partiels dont lapplication pose souvent des problémes relativement aux aspects qui ont été ignorés (par exemple, une solution technique peut s avèrer dangereuse pour I environnement et ou socialement inacceptable). La recherche interdisciplinaire a pour objectii de faire coopérer des approches disciplinaires de facon à compléter et à harmoniser les résultats sectoriels. puis à elaborer un point de vue synthétique. De plus, elles se caractérisent aussi par une forte prise en compte des interfaces, souvent abordées superficiellement dans les approches classiques, monodisciplinaires.

\section{L'environnement et ses dynamiques}

Rappelons d'abord que par environnement on entend " environnement de l'homme et des sociétés humaines - (Jollivet et Pavé, 1992, 1993) ${ }^{7}$. II se comprend à toutes les échelles d'espace, du local (l'environnement de l'individu ou du petit groupe, comme une communauté villageoise, à l'environnement de l'ensemble des sociétés humaines : l'environnement global ou planétaire). La définition que nous proposions était la suivante :

Lenvironnement est l'ensemble des milieux " naturels " ou * artificialisés - de l'écosphère où l'homme s'est installé, qu'il exploite, qu'il aménage, et l'ensemble des milieux non anthropisés nécessaires à sa survie. Ces milieux sont caractérisés :

- par leur géométrie, leurs composantes géophysiques, chimiques, biologiques, humaines et celles résultant de l'action de l'homme :

- par la distribution spatiale de ces composantes :

- par les processus de transformation, daction ou d'interaction, impliquant ces composantes et les faisant changer dans l'espace et dans le temps ;

- par leurs multiples dépendances par rapport aux actions de I'homme :
- par leur importance pour le développement des sociétés humaines.

Sans reprendre dans le détail l'argumentation développée à cette occasion, nous avons montré que les recherches sur l'environnement s'articulent autour de trois grands ensembles de questions, prenant progressivement valeur de paradigmes : - Lenvironnement : un grandes variables géophysicochimiques et biolo- champ de recherche en giques) :

ii) celui de la diversité biologique et des ressources renouvelables :

iii) celui du cadre et du mode de vie, de la santé des populations et sociétés humaines.

Ce dernier point est relatif aux aspects humains ; il to renvoie à la question du développement et des duprogramme modes de développement des sociétés humaines, Environnement du CNRS aux questions de l'action de l'homme sur la nature, à celles des solidarités entre les hommes.

6, 5-29.

On pourra également consulter :

Du point de vue scientifique, ce découpage iden- Godard O. (1995). tifie, très globalement, deux grands groupes de disci- - L'environnement, du plines : i) les sciences de la nature (sciences de la champ de recherche au
planète et sciences de la vie) et ii) les sciences de concept; une hiérarchie planète et sciences de la vie) et ii) les sciences de I'homme et de la société.

concept: une hiérarch
enchevêtrée dans la formation du sens $:$ Revue

Les trois composantes (cases) de la figure 4 sont en internationale de systémique relation ; trois couples de questions peuvent être 9,4,405-428. 
Tableau I. Exemples de programmes et d'institutions (organismes de recherche, agences, etc.) intervenant aux niveaux national et international dans les recherches sur l'environnement et/ou le développement.

Cette liste n'est évidemment pas exhaustive et forcément simplificatrice (par exemple, beaucoup d'organismes de recherche centrés sur l'environnement prennent déjà et nécessairement en considération des questions de développement et inversement). Par ailleurs, dans la nébuleuse des organisations non gouvernementales certaines contribuent, quelques-unes très positivement, d'autres moins, à cet effort de recherche".

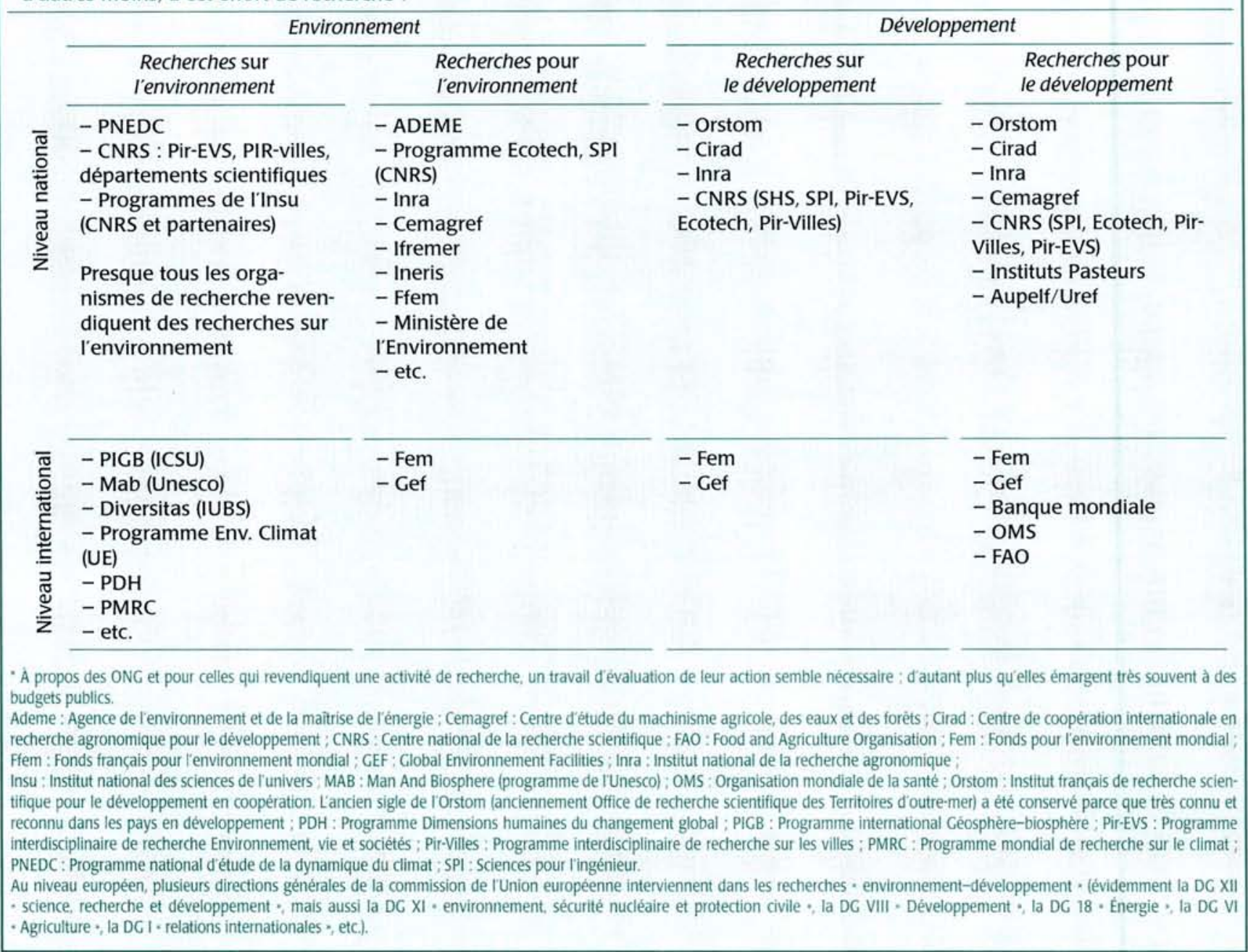

énoncés à propos des influences respectives de ces cases sur les deux autres. Par exemple la relation entre la case - cadre de vie, etc. ", elle-même explicitement liée aux sociétés et à leur développement, et la case " grands équilibres planétaires - suggère les deux questions suivantes :

i) Quel est le rôle des sociétés humaines et de leur développement dans les modifications des " grands équilibres planétaires - ?

C'est-à-dire distinguer, dans l'évolution de l'environnement, ce qui relève de processus naturels (ou spontanés) de processus d'origine anthropiques, tout en sachant qu'en plus ces deux types de processus peuvent interagir. Pour prendre un exemple maintenant bien connu, les émissions de $\mathrm{CO}_{2}$ dans l'atmosphère relèvent, d'une part, de processus naturels (les volcans et la respiration de nombreux êtres vivants en sont en partie responsables) et, d'autre part, de l'activité humaine (production d'énergie, transports).
Cette dernière partie devient de plus en plus importante depuis deux siècles. Comme le $\mathrm{CO}_{2}$ est un gaz à effet de serre, on craint et on commence à suspecter des modifications importantes des climats de la planète. Par ailleurs le $\mathrm{CO}_{2}$ est utilisé par les plantes par le biais du processus de photosynthèse : l'augmentation de la concentration atmosphérique de ce gaz peut-elle influer sur la croissance de ces plantes et comment ? Quels en seront les effets sur les écosystèmes, les agrosystèmes, les hydrosystèmes et en retour sur la composition de l'atmosphère ellemême ?

Question qui peut se renverser :

ii) Les modifications des grands équilibres planétaires influent-elles sur les sociétés humaines et sur leur développement?

On peut toujours prendre l'exemple du $\mathrm{CO}_{2}$ atmosphérique. On imagine bien que d'éventuels changements climatiques, consécutifs aux modifications de 
Figure 4. Trois grands ensembles de questions, dont la formulation prend progressivement valeur de paradigmes, fondent implicitement les recherches sur l'environnement. On notera cependant quá lidée de - grands équilibres ; notamment géophysicochimiques, se substitue progressivement celle de dynamiques planètaires - et de - viabilité - des systèmes (notamment des écosystèmes) plus conformes á la réalité de l'évolution permanente de notre environnement. constamment sur une trajectoire confinée dans un espace đ'état qui assure la viabilité de lécosphère et tout particulièrement la renouvelabilité des ressources de facon à ce que lhomme puisse vivre dans son environnement et en vivre. En termes de systèmes dynamiques, limportant est de se situer

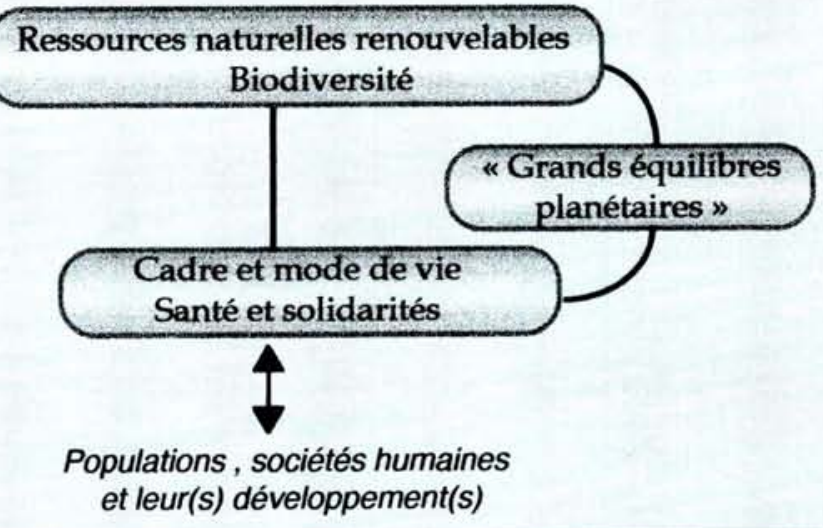

l'effet de serre suite à l'accumulation de ce gaz, auront des répercussions importantes au niveau régional, par exemple sur l'agriculture. De même et indirectement, la crainte de modifications climatiques devrait entraîner la mise en place de procédures et des techniques permettant la limitation des émissions de $\mathrm{CO}_{2}$ qui auront des conséquences économiques importantes (dues, en particulier, aux modifications des techniques de transports et de production d'énergie).

Bien que les principales approches relèvent classiquement des sciences de la nature, l'étude scientifique des questions posées demande obligatoirement une approche interdisciplinaire et implique ainsi d'autres secteurs scientifiques.

Par ailleurs, le nécessaire recours aux modèles et donc à la modélisation, implique les sciences mathématiques et informatique. Notons au passage que le modèle, parmi ses nombreuses fonctions, est un outil de dialogue entre disciplines et un instrument de synthèse interdisciplinaire (cf. figure 5). Cela lui confère un statut particulier dans la démarche scientifique actuelle ${ }^{8}$

Enfin, la progressive formulation des aspects humains met directement en rapport les problèmes d'environnement et les questions relatives aux sociétés humaines et à leur développement. Le lien est donc explicitement établi.

\section{Les sociétés humaines et leur(s) développement(s)}

Comme pour l'environnement, il serait souhaitable de définir le développement. Nous ne nous risquerons pas à un tel exercice et nous retiendrons seulement un cadre général :

Le développement des sociétés humaines correspond à une amélioration continue des conditions de vie de l'ensemble des individus de ces sociétés (santé, sécurité, cadre de vie, travail et emploi, éducation et culture), bien qu'à des degrés divers et suivant des modalités différentes. Cet objectif s'accompagne d'une nécessité de croissance économique et de progrès technologique ${ }^{9}$. II doit s'inscrire dans un temps long, celui de plusieurs générations humaines ; c'est pourquoi on parle maintenant de développement durable.

En pratiquant ensuite une analyse analogue à celle faite pour l'environnement, en tenant compte de la liste de 51 indicateurs choisi par la Banque mondiale (1992, voir tableau II) mais pas exclusivement, on peut proposer que, dans son expression actuelle la question du développement s'articule autour de quatre préoccupations : i) celui de la croissance économique ; ii) celui du * contrôle * de la démographie : iii) celui du progrès technologique ; iv) celui du bien être individuel et du progrès social (éducation, culture, travail et emploi, santé et sécurité).

On remarquera au passage que beaucoup d'indicateurs de la Banque mondiale, 51 répartis en 16 thèmes, sont économiques ( 23 sur 51 ), que nombre d'entre eux sont très corrélés ( $\mathrm{Paz}$ et $\mathrm{al}, 1993)^{10}$ et qu'aucun ne fait explicitement référence au progrès technologique (voir Quels indicateurs?). Cette dominante des composantes économiques n'est pas nouvelle. On peut même dire que les recherches sur le développement ont d'abord concerné cette discipline. On notera, par exemple, que, dans son édition de 1980, I'Encyclopaedia Universalis présente l'approche scientifique des questions de développement en faisant référence à trois disciplines : l'économie, la sociologie et le droit ; l'économie fut, historiquement, en première ligne. Aujourd hui et singulièrement avec le concept de développement durable, bien d'autres secteurs scientifiques sont concernés.

Par ailleurs et bien que ces indicateurs aient été conçus dans l'idée actuelle du développement durable, on ne trouve aucune trace d'indicateur relatif à l'environnement (sauf peut-être une référence à l'urbanisation). Cela étant, cette construction ne traduit pas exactement les dynamiques scientifiques ${ }^{11}$ et celles exprimées par l'autres organisation comme le Pnud. Une volonté de rapprochement des problématiques et des points de vue scientifiques sur l'environnement et le développement est de plus en plus clairement exprimée. Une réflexion sur la conception d'indicateurs de développement durable est menée activement au niveau international ${ }^{12}$.

De fait, dans l'idée du développement durable, le * point d'entrée environnement - apparaît clairement par le truchement des notions de ressources renouve-
${ }^{8} \mathrm{Cf}$. par exemple Pavé A. (1994).

- Modélisation en biologie et en écologie - Aléa, Lyon. - Tendances nouvelles en modélisation pour l'environnement :, Actes des Journées du PIR-EVS, Paris, janvier 1996 (4 volumes).

${ }^{9}$ Du moins, est-ce l'idée la plus fréquemment admise ! ${ }^{10}$ Paz B., Degang J., Le Boulengé E. (1993). - Quelle classification pour les pays en voie de développement? : mémoire, université catholique de Louvain.

"Par exemple, la conférence internationale

- Écologie, sociologie, économie - (op. cit.) avait dans ses préoccupations de faire converger les approches de ces disciplines.

${ }^{12}$ Lors du colloque international - Quel environnement au $\times x^{e}{ }^{e}$ siècle ? Environnement long terme et démocratie (op. cit.), un atelier a été consacré à ce sujet. Cependant à travers les contributions présentées. on notera les progrès méthodologiques nécessaires pour donner une solution acceptable et opératoire au problème des indicateurs du développement durable. 
Tableau II. Indicateurs du développement

(Banque mondiale : Rapport sur le développement dans le monde, Oxford University Press, 1992)

Indicateurs de base

1. Pays

2. Population (millions d'habitants)

3. Superficie (milliers de $\mathrm{km} 2$ )

4. Densité de la population

5. PNB par habitant (en dollars)

6. PNB par habitant, croissance annuelle (\%)

7. Inflation annuelle moyenne (\%)

8. Espérance de vie à la naissance (années)

9. Analphabétisme (adultes, \%)

Croissance de la production (\%)

10. Croissance moyenne annuelle du PIB

11. Croissance moyenne annuelle de l'agriculture

12. Croissance moyenne annuelle de l'industrie

13. Croissance moyenne annuelle de la manufacture

14. Croissance moyenne annuelle des services

Structure de la production

15. PIB par habitant

16. Répartition du PIB (\%) dans l'agriculture

17. Répartition du PIB (\%) dans l'industrie

18. Répartition du PIB (\%) dans la manufacture

19. Répartition du PIB (\%) dans les services

Agriculture et alimentation

20. Valeur ajoutée par habitant dans l'agriculture

21. Importation de céréales par habitant

22. Aide alimentaire en céréales par habitant

23. Consommation d'engrais

24. Indice moyen de production alimentaire par habitant

Structure de la demande

25. Répartition du PIB (\%) dans la consommation des administrations publiques

26. Répartition du PIB (\%) dans la consommation privée

27. Répartition du PIB (\%) dans l'exportation des biens et services

Dépenses de l'administration centrale

28. Dépenses totales (\%) en défense (\%)

29. Dépenses totales $(\%)$ en éducation (\%)

30. Dépenses totales (\%) en Santé (\%)

31. Dépenses totales (\%) en habitat et équipements collectifs, sécurité sociale et aide sociale

32. Dépenses totales (\%) en services économiques

lables et non renouvelables dans les modèles économiques ( $c$. par exemple, Willinger, 1996) ${ }^{13}$. II est classiquement intégré dans le discours sur le développement durable d'origine plutôt écologique ou environnementaliste. Ainsi Di Castri propose-t-il les " quatre pieds de chaise - du développement durable : culturel, social, économique et environnemental (Di Castri, 1995) ${ }^{14}$; tout déséquilibre se traduit par une chaise bancale et donc un développement
33. Dépenses totales (\%) diverses

34. Dépenses totales (\%) du PNB

35. Rapport global excédent/déficit en \% du PNB

Recettes courantes de l'administration centrale

36. Recettes courantes en \% du PNB

Structures des importations des marchandises

37. Importations de marchandises : produits alimentaires $(\%)$

38. Importations de marchandises : produits industriels (machines) $(\%)$

Structures des exportations des marchandises

39. Exportations de marchandises : combustibles, minéraux et métaux (\%)

40. Exportations de marchandises : autres produits primaires (\%)

Apports de l'aide publique au développement (ADP)

41. Décaissements nets d'ADP de toutes origines par habitant (en dollars)

Dette extérieure totale

42. Dette extérieure totale (en millions de dollars) Accroissement passé et projeté de la population

43. Taux annuel moyen d'accroissement (\% de la population totale)

Démographie et fécondité

44. Taux brut de natalité (pour 1000 habitants)

45. Taux brut de mortalité (pour 1000 habitants)

Santé et nutrition

46. Apport calorique journalier par habitant

Éducation

47. Pourcentage de la classe d'âge pertinente d'inscriptions dans l'enseignement primaire

48. Pourcentage de la classe d'âge pertinente d'inscriptions dans l'enseignement secondaire

49. Taux net de scolarisation primaire

Urbanisation

50. Pourcentage de la population urbaine

51. Taux de croissance (annuel) de la population urbaine

dysharmonieux. Cette métaphore pose implicitement le problème de l'utilisation et de la distribution des richesses. On notera que le progrès technologique n'est pas non plus explicite dans cette représentation, de même les questions de santé qui sont probablement intériorisées dans le développement social. Cela étant, l'image est très séduisante.

Plus généralement, si la transmission du savoir est bien précisée (éducation), si le progrès technologique 


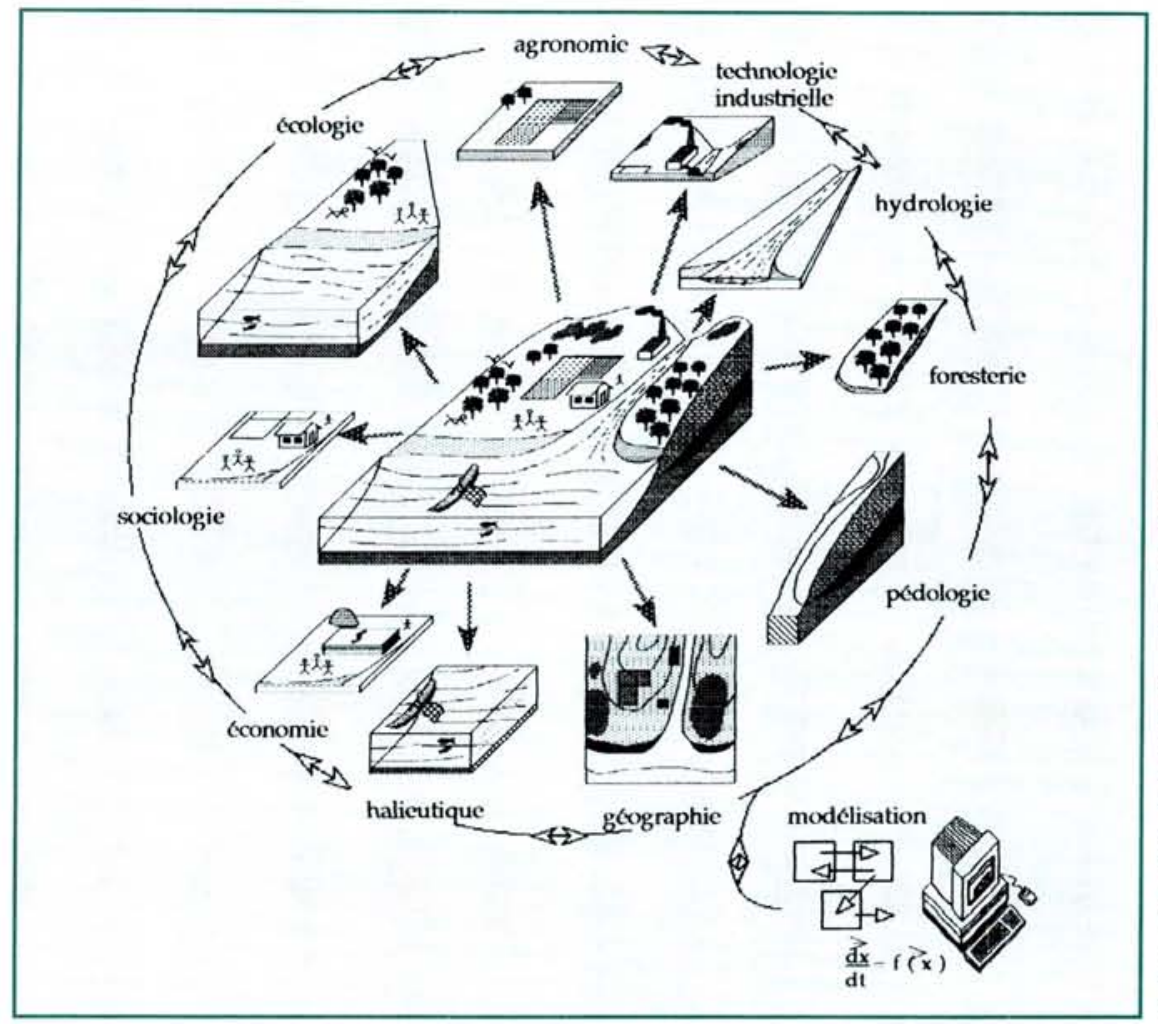

Figure 5. La modelisation intervient comme méthode dans la plupart des approches disciplinaires. Cest aussi un facteur d unification dans te dialogue interdisciplinaire. Le modéle est lobjet qui concrêtise le point de vue commun sur la situation ètudiée : a ce titre, il est média. teur et instrument de discussion. Pat ailleurs, outil de synthèse il peut aider ou guider la prise de dècision. En tout êtat de cause sa construction et son utilisation demandent des specialistes avertis. Enfin, il peut aussi devenir moyen de manipulation, au sens - social - du terme II y a lieu de ètre conscient et averti de ce risque et de promouvoir une - déontologie - relative à la pratique de la modélisation et a l usage des modèles

est souvent pris en compte implicitement, en revanche le développement du dispositif d'acquisition, de formulation et d'organisation des connaissance, c'est-àdire la recherche scientifique, n'est pas explicité. Pourtant, on s'accorde à dire que cet aspect est stratégique et jouera sans doute un rôle déterminant dans l'avenir : la capacité des sociétés à acquérir des connaissances nouvelles, a intégrer celles obtenues par les autres et à utiliser à leur bénéfice ces ensembles de connaissances.

La recherche scientifique est non seulement un des objectifs et un des moyens du développement, voire une condition nécessaire : c'est aussi une approche des problèmes de développement et tout particulièrement de ceux du développement durable. À cette fin, cette recherche peut se fonder sur une nouvelle formulation des paradigmes, mieux adaptée à l'étude du développement durable, intégrant la dimension environnementale, comme on vient de le voir, absente ou quasiment de la problématique du développement. Ainsi et dans une deuxième approximation on peut esquisser les perspectives suivantes : i) croissance économique et distribution des richesses ; ii) évolution démographique et sa maitrise ; iii) bien être individuel et progrès social ; iv) progrès technologique et scientifique ; v) renouvelabilité des ressources reproductibles - environnement viable.

Le glissement des termes est lui aussi significatif : - Lidée de croissance économique est de plus en plus accompagnée du souci de la distribution des richesses,

\section{Quels indicateurs ?}

Des indicateurs globaux, comme l'IDH, indicateur du développement humain, sont très réducteurs et traduisent mal les aspects qualitatifs. L'IDH est utilisé par le PNUD (Programme des Nations unies pour le développement), qui en a đ'ailleurs, lui-même, une vision critique. On pourra consulter :

- Rapport mondial sur le développement - (Programme des Nations unies pour le développement, ed.), Economica, 1996, 251 p.

Dans ce rapport on trouvera également les indicateurs du développement choisis par le PNUD, plus nombreux et moins

économico-centrés que ceux de la Banque mondiale ( 48 tableaux thématiques avec une moyenne de huit indicateurs par tableau), dont l'IDH est la synthèse partielle. Certains d'entre eux explicitent très clairement les soucis relatifs à l'environnement et à la gestion des ressources naturelles. Lanalyse de ces thèmes et indicateurs va dans le sens d'une catégorisation en 4 (figure 6), puis cinq grands ensembles (figure 7) de préoccupations qui peuvent structurer un champ de recherche sur le développement avec une prise en considération explicite des problèmes environnementaux et de ceux de la gestion des ressources naturelles. On notera également, dans cette mème publication, trois contributions importantes :

- Léquité intergénérationnelle, oui, mais qu'en est-il de l'équité aujourd'hui ? - par Robert M. Solow.

- Humaniser la croissance : la voie de l'équité -, par Fernando Henrique Cardoso.

- Le défi et l'exemple de l'Afrique du Sud *, par Nelson Mandela. 


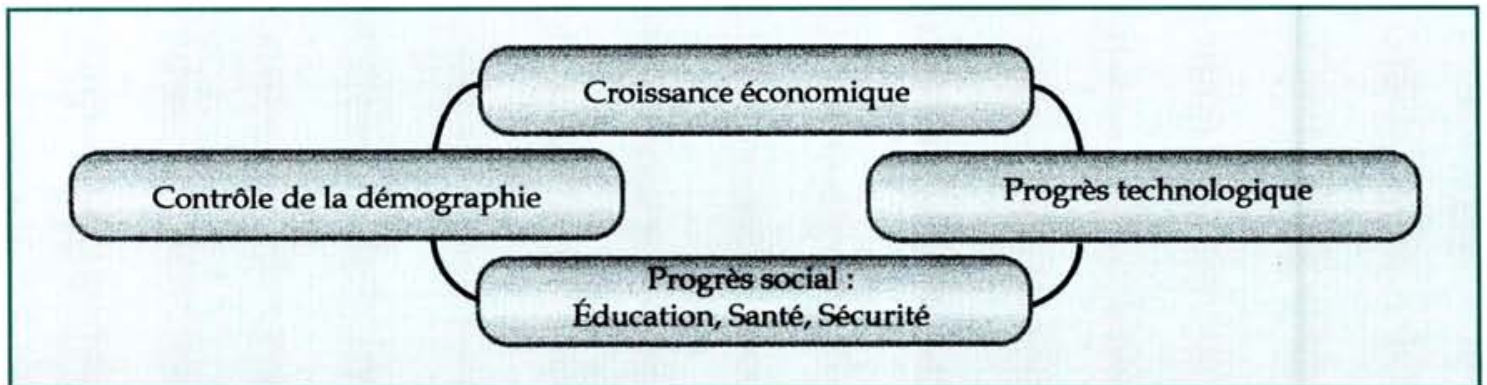

Figure 6. Recherches sur le développement et leurs préoccupations telles qưelles peuvent ètre dégagées des indicateurs du développement lđaprès les indicateurs de la Banque mondiale, 1991) et des tendances actuelles de la recherche. De la même façon que pour lenvironnement, derrière ces préoccupations des paradigmes. pas toujours explicites, conditionnent les recherches.

dans le sens d'une répartition * plus juste - à l'intérieur des diverses sociétés et entre celles du Nord et celles du Sud.

- On sait maintenant que la démographie n'est pas réductible à un processus explosif mais répond à des dynamiques plus subtiles. On peut donc substituer le terme plus général d'évolution à celui d'explosion. L'enjeu reste néanmoins de maîtriser cette évolution et ses conséquences.

- Le progrès technologique ne correspond pas uniquement à la découverte de nouvelles technologies mais aussi au transfert et à l'adaptation technologiques. Par exemple, l'adoption de la glacière par la pêche piroguière en Afrique de l'Ouest a considérablement modifié la pratique des pêcheurs et l'économie de la pêche. De surcroît et comme il a été signalé ci-dessus, au progrès technologique il faut aussi associer le progrès scientifique avec la même idée : le progrès n'est pas réductible à l'acquisition de connaissances nouvelles, au niveau international, mais à l'accès aux connaissances, à leur appropriation et à leur transfert.
- Le progrès social n'est pas réductible à des considérations sécuritaires, sanitaires ou d'instruction, mais prend d'autres dimensions avec la recherche de solidarités et d'expressions culturelles nouvelles. Ce dernier point est en plein débat : comment intégrer la diversité culturelle, les conditions locales de vie, dans une éducation devant aussi transmettre des notions universelles ?

- La prise en compte de la renouvelabilité des ressources et de l'accessibilité à des ressources potentielles est également un progrès dans l'appréhension des relations entre l'homme et son environnement. Ce dernier n'est plus vu comme une source inépuisable de biens et de richesses, ou comme une poubelle sans fond, mais comme une entité dont il faut préserver la viabilité pour assurer le renouvellement des ressources (air, eau, aliments, etc.) ou bien le potentiel par une exploitation raisonnée des ressources non renouvelables.

II n'est cependant pas inutile de préciser que la transition entre les deux schémas : de celui du déve-
Figure 7. Proposition de para digmes pour la recherche sur le développement durable. Apparaissent explicitement des relations avec l'environnement. Précisons a nouveau que la viabilité de l'environnement, expression quelque peu équivoque, correspond à tidee que thomme puisse vivre (bien) dans cet environ. nement et en vivre (bien). En sachant que ce critère, très anthropocentré, peut évoluer au cours du temps, mais qu'il suppose néanmoins que certaines propriétés et fonctions soient maintenues latmosphère respirable, eau consommable, ècosystèmes fonctionnels assurant la reproductibilité des ressources vivantes, etc.) ou même améliorées.

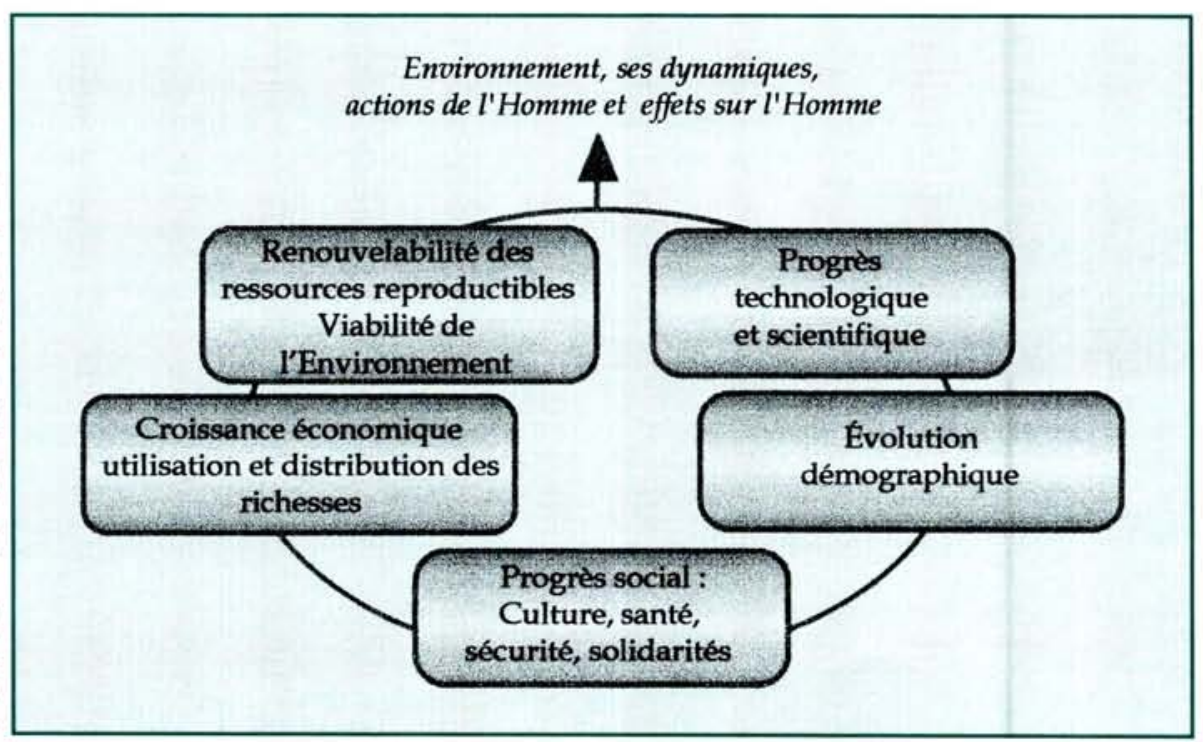




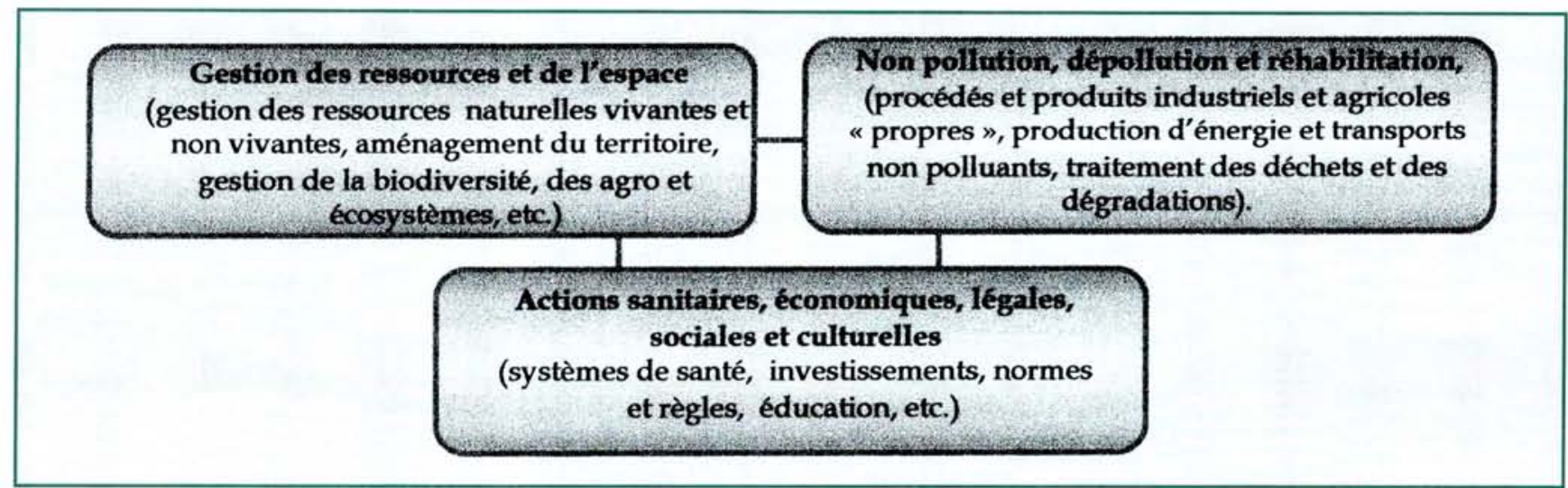

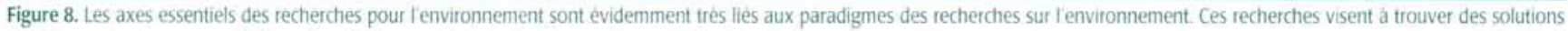
techniques ou autres aux problemes denvironnement et à etudier les conséquences de leur mise en œuvre

loppement à celui du développement durable, n'est pas encore pleinement acquise (c'est le moins qu'on puisse dire). Par exemple, la vision étroitement économiste de la société, notamment dans son acception extrême et ultralibérale de l'école de Chicago, s'oppose clairement à cette conception. Or, cette conception du développement fait évoluer les concepts et modèles économiques eux-mêmes (qui deviennent moins schématiques et moins simplistes) et intègre d'autres dimensions non traduites (voire non traductibles) en termes purement économiques.

Sur la base d'un schéma du type de celui présenté dans la figure 7 , on peut imaginer comment organiser des actions de recherche qui permettent d'étudier les mécanismes fondamentaux intervenant dans chacune de ces boîtes et surtout de préciser leurs interrelations. Ces dernières permettent de construire un questionnement du type proposé pour la recherche sur l'environnement. On peut également se servir de ce genre de repère, dans une version plus détaillée, pour l'analyse des causes de * dysfonctionnement ". C'est-à-dire d'identifier les raisons d'une dérive des objectifs de développement tels qu'ils sont élaborés ou adaptés.

II est bon de le souligner encore, on voit clairement les rapports à l'environnement par l'intermédiaire de la renouvelabilité des ressources reproductibles (en l'occurrence des ressources vivantes) et de la notion plus générique d'environnement viable, des références au bien-être, donc à la santé, elle-même très liée à l'état de l'environnement et des progrès scientifiques et technologiques (mieux comprendre notre environnement, ses dynamiques pour mieux décider et agir).

Enfin, la notion même de développement et plus encore celle de développement durable impliquent une approche spatiale et temporelle des phénomènes, à savoir une étude de la dynamique des systèmes spatialisés, qu'ils soient naturels, techniques ou sociaux.

\section{Les recherches pour l'environnement, les recherches pour le développement}

Le processus d'acquisition des connaissances sur l'environnement et le développement doit être complété par l'étude des conditions concrètes d'intervention de I'homme afin de préserver ou d'améliorer son environnement et, simultanément, de faire progresser ses conditions de vie.

Les recherches pour l'environnement sont évidemment très liées aux recherches sur l'environnement. Elles ont pour objectif :

- de trouver des solutions techniques, réglementaires, économiques et sociales permettant de réhabiliter les environnements dégradés, d'éviter les dégradations futures, de gérer les ressources et l'espace, d'assurer un bon état sanitaire par un bon environnement :

- de prévoir les conséquences directes ou indirectes de l'application de ces solutions. Comme nous l'avons déjà souligné, ces recherches relèvent principalement des sciences de l'ingénieur, mais impliquent également des sciences sociales (sociologie, économie, pédagogie, droit, etc. $)^{15}$.

La recherche scientifique ne peut pas, ou ne peut plus, faire l'économie de l'étude des actions de I'homme sur son environnement et pour son environnement. A l'image des sciences de l'ingénieur, il ne s'agit pas d'une stricte application des connaissances de base mais d'une véritable recherche des conditions de mise en œuvre de ces connaissances.

Dans une première lecture, les recherches pour le développement pourraient concerner toutes ou presque toutes les activités de recherches scientifiques et technologiques. Par exemple, les travaux sur la fusion thermonucléaire contrôlée relèveraient à l'évidence d'un souci de développement et même de développement durable pour assurer des ressources énergétiques quasiment inépuisables pour l'humanité. Cependant, ce n'est pas ce qui est implicitement entendu lorsqu'on fait référence aux recherches pour ${ }^{15}$ Pavé A., Jollivet $M$., le développement. En effet, on s'intéresse à résoudre op. cit. 


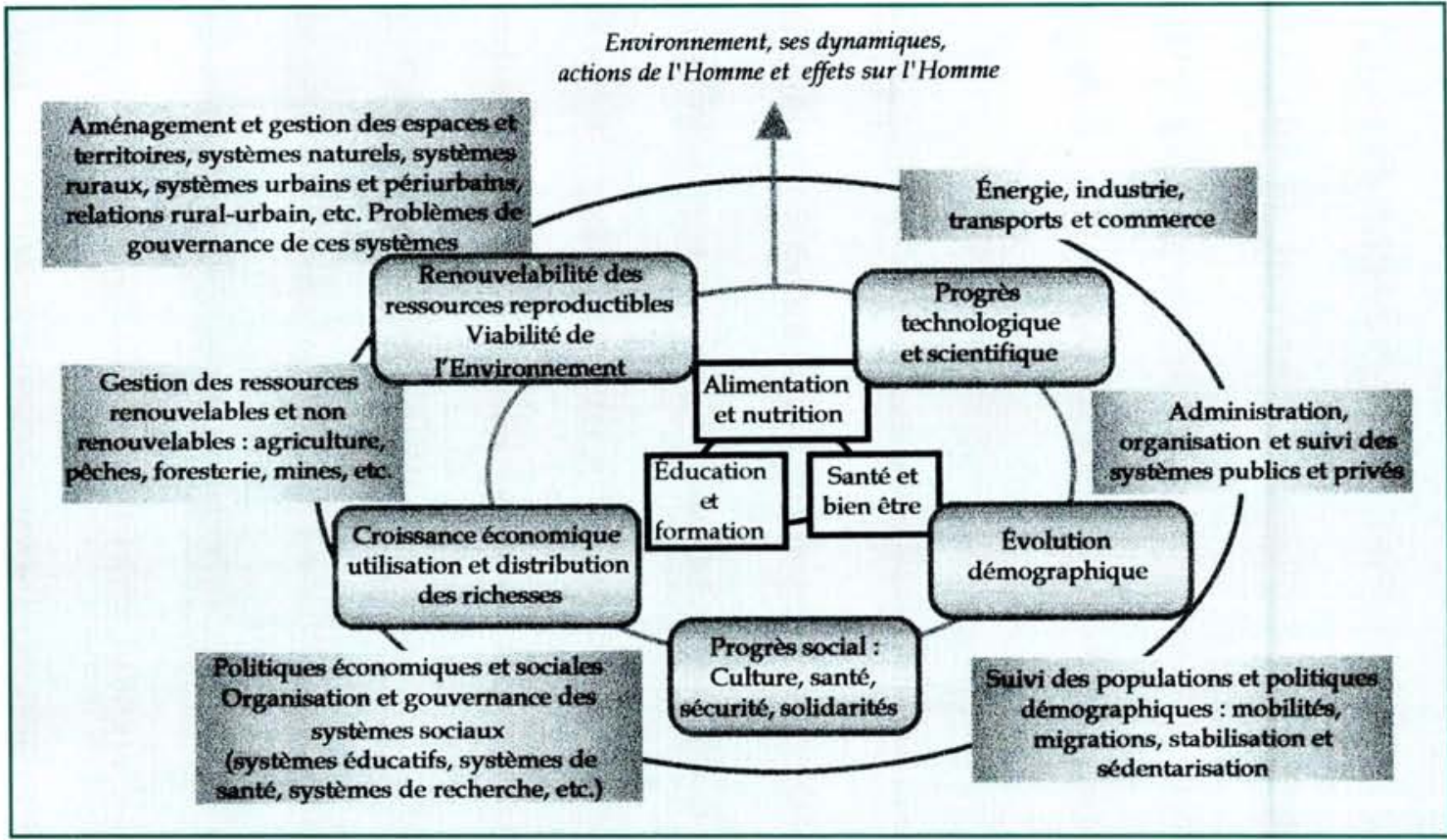

16 Par exemple, le Cimpa (Centre international de mathématiques pures $\mathrm{e}$ appliquées) s'intéresse à la formation mathématique et informatique des cadres des Ped, notamment africains. La lecture des programmes montre qu'on y fait des mathématiques d'un très bon niveau, voire très pointues. Si on est parfaitement convaincu que dans tous les pays du monde on peut former et trouver d'excellents mathématiciens, les mathématiques en questions sont-elles concrètement - utiles pour le développement II ne s'agit pas de bouder les mathématiques avancées, mais de ne pas faire que celles-ci. On ne peut qu'être attentif a l'évolution actuelle de ce centre et à la réflexion qui

la sous-tend qui vise a revoir ce schéma, tout particulièrement dans un contexte scientifique et technique oủ la

modélisation devient une méthode de plus en plus répandue.

Figure 9. Les recherches pour le développement visent daabord á satisfaire les - besoins élémentaires · (partie centrale : alimentation et nutrition, santê, éducation et formation), ensuite à examiner concrètement les conditions d'un développement durable (partie externe). L'ensemble s'organise autour des paradigmes đe la recherche sur le développement. De cet ensemble émerge une série de questions mettant en relation les divers éléments de ce schéma tout en notant bien que ce demier, comme les précédents, na aucune prétention à l'exhaustivité. II na de valeur quillustrative pour montrer une dèmarche possible de problèmatisation et dorganisation du questionnement.

les problèmes de dysfonctionnement que subissent les sociétés du Nord, à imaginer des schémas plus soucieux de l'homme et de la nature et surtout à tenter de combler les écarts entre ces sociétés et celles du Sud par des recherches et actions spécifiques concernant ces dernières. Dans ce contexte les relations entre environnement et développement prennent aujourd hui un relief particulier.

Les relations entre pays dits " développés - et pays dits " en développement " ont un lourd passé : l'époque coloniale et l'idéologie de la - mise en valeur ", l'époque post-coloniale, quelquefois néocolonialiste, avec les tentatives de transferts de modèles du Nord (des transferts technologiques aux modèles de développement économiques et sociaux) et les nombreux échecs de ces tentatives de transfert ou, ce qui est plus rassurant, leur récupération à des fins inattendues ou encore leur intégration dans un développement autonome. Par exemple les tentatives d'industrialisation de la pêche maritime en Afrique de l'Ouest n'ont pas empêché le développement de la pêche piroguière (dite * artisanale *). Cela étant, de grands progrès ont été enregistrés essentiellement sur les questions fondamentales de subsistance, sur la satisfaction des besoins essentiels, à savoir l'alimentation (quantitativement et qualitativement), la santé, l'éducation et la formation.

Dans ces trois cas et plus particulièrement pour les deux premiers, le rôle de la recherche a été déterminant :

i) Pour l'alimentation avec le développement d'une agriculture adaptée, sur la base d'une recherche agronomique spécifique, même si beaucoup reste à faire notamment par la promotion d'une agriculture productiviste respectueuse de l'environnement. Les autres ressources, notamment halieutiques et aquacoles, sont aussi l'objet d'une recherche et de développements technologiques actifs.

ii) Pour la santé avec les succès indéniables d'une médecine tropicale efficace, même si de nombreux progrès restent à faire sur certaines grandes endémies (paludisme, sida, bilharziose). En revanche, la mise en place de systèmes de santé adaptés pose encore de nombreux problèmes : l'intégration du médical, du socioculturel et des aspects économiques reste à faire. iii) Pour l'éducation et la formation, le schéma a le plus souvent consisté à copier ou à transférer les modèles éducatifs sans une recherche de contenus et de modes de transmission du savoir adaptés. Sans ressortir l'exemple éculé de " nos ancêtres les Gaulois ", mais qui fut bien réel, on est étonné de l'intervention de certaines associations ou de certains organismes ${ }^{16}$. À une époque ou la maîtrise des savoirs et des connaissances est de plus en plus considérée comme une des clés, sinon la clé, du développement il y a lieu de porter une attention particulière à l'éducation et à la formation et à rechercher les voies adaptées économiquement, socialement et culturellement. II ne s'agit pas non plus de faire dans le particularisme outrancier mais de maintenir un fonds commun de savoir entre les hommes qui ménage les spécificités et les originalités.

Les dimensions économiques et technologiques sont omniprésentes :

- En arrière plan, par exemple elles sont de plus en plus prises en compte par les chercheurs des sciences 


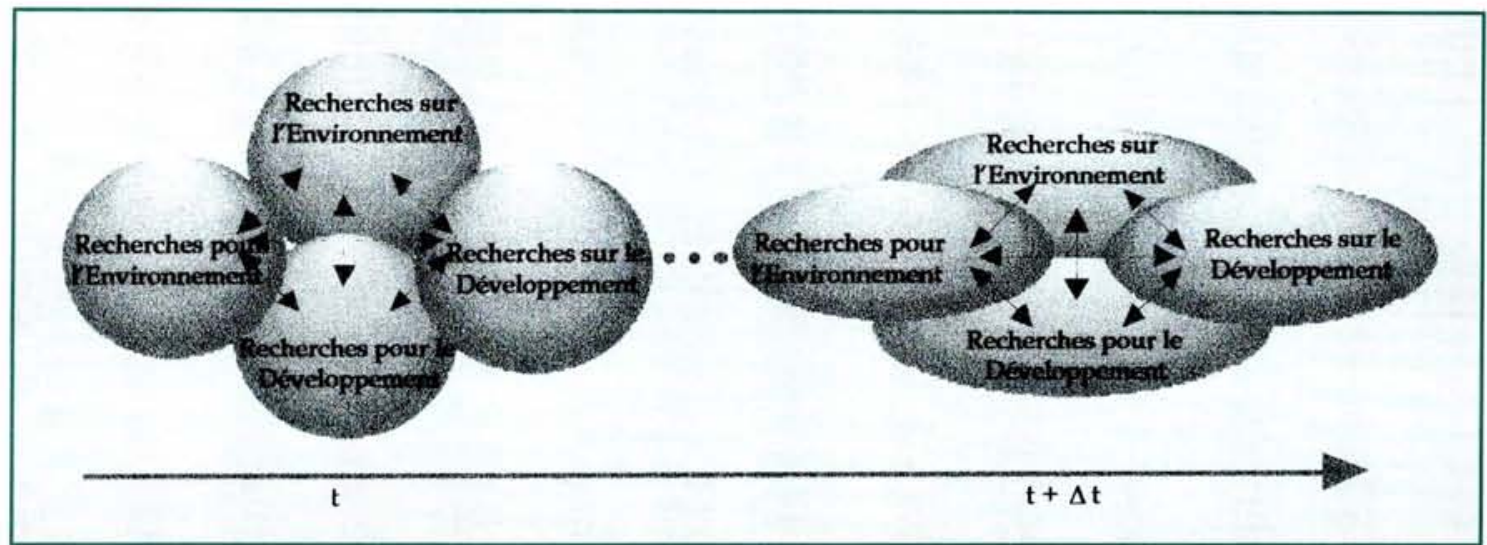

Figure 10. Les interactions entre champs de recherche devraient conduire à une coévolution de ces champs, à la modification de leurs contours, à une plus étroite imbrication de leurs travaux et à une meilleures adaptation de leurs paradigmes, sans pour autant quils perdent leurs identitès.

de la nature eux-mêmes qui tentent de trouver des solutions technologiquement et économiquement viables dans le contexte des PED. II se peut même que la considération de ces contraintes conduisent à des solutions meilleures que celle utilisées " classiquement $=17$.

- De façon plus profonde, l'étude des économies et des technologies telles qu'elles s'expriment et se conçoivent sur le * terrain ", au-delà des modèles théoriques normatifs pensés dans les laboratoires du Nord, prend de plus en plus d'importance (économie anthropologique, économie et écologie, confrontation des modèles théoriques avec les réalités de terrain, études de technologies alternatives, etc.). II s'agit d'un secteur actif, intellectuellement pertinent et concrètement utile.

Enfin, tout ce qui concerne les questions d'organisation des systèmes sociaux et des systèmes de production, d'administration, de politiques, d'aménagement et de gestion des espaces et territoires (notamment à travers la question de l'urbanisation, de gestion des systèmes ruraux et naturels), de gestion des ressources naturelles, renouvelables ou non, et enfin des migrations des populations humaines font l'objet de recherches très actives.

Ces recherches sont lancées soit à l'initiative de la communauté scientifique elle-même, soit à la suite d'interrogation par divers acteurs du développement. On voit qu'elles recoupent très précisément nombres de recherches pour l'environnement citées ci-dessus.

\section{Environnement et développement}

\section{Viabilité, durabilité, acceptabilité. Une niche écologique pour la coévolution des approches scientifiques de l'environnement et des recherches au service du développement}

Les schémas et paradigmes proposés sont évidemment très sujets à discussions et à débats. Ils deman- dent certes à être précisés. Néanmoins, on voit très clairement leurs complémentarités, leurs convergences, mais aussi leurs points de grippage, les tensions possibles. Par exemple, au sujet des milieux naturels, la position environnementaliste tend plutôt vers la protection de la nature (par exemple, pour préserver la biodiversité). En revanche, la position développementaliste aura tendance à privilégier l'exploitation, la valorisation, l'aménagement du milieu naturel. Sur un autre exemple, celui de la démographie, on a vu à l'occasion de la conférence du Caire s'affronter nettement ces deux positions, les environnementalistes dans leur expression extrême prônant une diminution drastique de la population mondiale, les développementalistes se satisfaisant d'une croissance démographique en faisant l'hypothèse (raisonnable) que les processus endogènes de régulation allaient bientôt stabiliser cette population $^{18}$. De fait, il faut faire évoluer les opinions, en particulier celles largement répandues de - développement comme atteinte à l'environnement * ou * d'environnement comme frein au développement * en - développement comme moyen d'améliorer l'environnement " ou comme * environnement moyen du développement (durable) :

Du point de vue scientifique, les recherches sur l'environnement, les recherches sur le développement et les recherches pour le développement ont avantage à se confronter et à se compléter ; en réalité la dynamique est déjà enclenchée ${ }^{19}$. Néanmoins, il faut savoir que ni l'un ni l'autre des champs scientifiques ne sortira indemne de cette confrontation : paradigmes, méthodes et théories changeront, évolueront. En ce sens là, on peut parler de coévolution à l'image de celle de deux ou plusieurs espèces biologiques. Dans certains cas l'interaction entre ces espèces (respectivement, entre ces champs scientifiques) conduit à une modification profonde de leurs caractéristiques propres, modifications, qui se traduisent par une meilleure adaptation globale de ces ensembles d'espèces (respectivement de ces champs scientifiques) à leur milieu et aux variations de celui-ci (respectivement aux questions posées, qu'elles soient d'origines endogènes, internes à la science, ou exogènes provenant d'autres acteurs sociaux). Enfin, chacune de ces espèces (respectivement de ces
${ }^{17}$ Par exemple, une équipe du Cirad, travaillant sur les technologie des lipides, a adapté un mode d'extraction de l'huile des fruits du palmier à huile fournissant un produit de bien meilleure qualité, notamment en contenu vitaminique, et moins coûteux que le procédé thermique classique.

${ }^{18}$ On trouvera une analyse de cette question dans:

Keifitz N. (1994)

- Demographic discord : The sciences,

$34,5,21-27$ Le Bras H. (1994). Les limites de la planète. mythes de la nature et de la population. Flammarion. Paris.

9 La conférence internationale - Écologie sociologie, économie * déjà citée est un exemple d'une telle dynamique. 
${ }^{20}$ Viabilité, durabilité et acceptabilité (acceptabilité au sens social) nous semblent complémentaires. Le mot viabilité peut être pris en référence à la théorie de la viabilité de J.-P. Aubin : on peut le réserver à l'environnement. Le terme de durabilité nous semble mieux adapté au développement. Les deux devraient être liés à la contrainte d'acceptabilité (sociale). Par exemple, un développement durable fondé sur un environnement viable peut être socialement inacceptable. On peut imaginer un environnement durable qui ne soit pas acceptable, ni même viable : d'ailleurs, la notion d'environnement durable a-telle même un sens ? Etc. Pa analogie avec la - chaise du développement durable - de F. Di Castri (op. cit.), viabilité durabilité et acceptabilite nous paraissent constituer le trépied sur lequel devraient se fonder les conditions, les moyens et les politiques de développement, ainsi que recherches scientifiques associées permettan d'identifier, voire de définir ces conditions, de concevoir ces moyens et d'envisager les termes de ces politiques.

Pour la théorie de la viabilite et son utilisation, on pourra consulter

Aubin J.-P. (1990). - A survey of viability theory $\cdot$ SIAM J. on Control and Information

28, 749-788.

Aubin J.-P. (1996).

- Une métaphore

mathématique du principe de précaution .

Natures, Sciences, Sociétés 4, 2, 146-154.

${ }^{21} \mathrm{Cf}$., par exemple, Bousquet F., Cambier C., Mullon C. Morand P., Quensière J. Pavé A. (1993). * Simulating the interaction between a society and a renewable resource -. J. Biol. Systems 1 ,

2 199-214

\section{Interactions et évolution}

Pour illustrer cette nécessité d'évoluer, on parle souvent de - modèle de la reine rouge *, image prise par Van Valen, inventeur du concept de coévolution. II se réfère par là au célèbre roman de Lewis Caroll, De l'autre côté du miroir oủ la reine rouge explique à Alice que les gens autour d'elle courent, tout se déplace, il faut donc aussi courir pour maintenir sa position par rapport à eux c'est-à-dire, en quelque sorte - pour rester sur place - Dans la niche écologique d'une espèce, les principales composantes sont les autres espèces qui interagissent avec elle. Ces autres espèces, changent, s'adaptent, évoluent ; donc l'espèce en question doit suivre la course en évoluant aussi (en changeant, en s'adaptant), sous peine de disparaître. La transposition scientifique se comprend : un champ scientifique, par exemple une discipline, doit tenir compte de l'évolution globale des autres disciplines, elle doit elle-même évoluer sous peine de se trouver en grande difficulté. D'une certaine façon les problèmes de la systématique biologique peuvent illustrer cette situation : trop fermée sur elle-même, n'intégrant pas ou peu les progrès des autres sciences du vivant, ne faisant pas évoluer ses paradigmes, elle s'est retrouvée isolée, en difficulté. Gageons que la prise de conscience actuelle va lui permettre de rattraper son - retard évolutif -. En revanche, l'écologie a su magistralement utiliser ce paradigme interne de la reine rouge et intégrer les progrès des autres disciplines. Plus généralement, les champs de recherche évoqués ici, ont intérêt à coévoluer, non seulement pour préserver leur propre existence, mais aussi et surtout pour résoudre des problèmes de grande importance pour les sociétés humaines actuelles et futures. Pour les lecteurs curieux, ils trouveront dans les bons ouvrages d'écologie une présentation détaillée de ce concept et de nombreux exemples et contre-exemples. Citons, en particulier :

Barbault R. (1992). Écologie des peuplements. Masson, Paris.

Combes C. (1995). Interactions durables. Masson, Paris.

recherches) doit évoluer pour rester dans la course, sous peine d'être dépassée et de risquer l'extinction, la disparition. II faut certes se garder de faire une transposition brutale, trop * naturaliste ", de ce concept écologique dans le champ de la recherche. Néanmoins l'image nous semble intéressante (voir Interactions et évolution).

Cependant, la coévolution entre espèces nécessite une " interaction durable " entre celles-ci. Toutes proportions gardées, gageons que pour des champs scientifiques c'est la même chose et que recherches sur le développement, recherches pour le développement et recherches sur et pour l'environnement doivent interagir durablement. Enfin, coévolution ne signifie pas fusion : chaque champ doit conserver son identité, son originalité, sa spécificité et sa dynamique propre (figure 10).

Enfin, le couplage fort, quasiment consubstantiel, entre environnement et développement (comme nous l'avons déjà fortement souligné : les sociètés humaines se développent en agissant sur leur environnement, d'une certaine façon l'environnement est un " produit - du développement) incite encore plus impérativement au renforcement des relations puis à la coévolution entre les champs de recherche. De ce point de vue un cadrage très général du genre * environnement et développement : viabilité, durabilité et acceptabilité ${ }^{20}$ nous paraît avoir un pouvoir fédérateur. Là encore, la modélisation devrait jouer un rôle de premier plan en facilitant le dialogue entre les champs de recherche, notamment en promouvant la construction de modèles qui assemblent dynamiques naturelles et dynamiques socioéconomiques ${ }^{21}$. Enfin, une organisation et une programmation scientifiques, si elle sont bien conçues, pourraient faciliter les rapprochements ou les organiser harmonieusement (voir Modèles de dynamiques).

\section{Modèles de dynamiques}

Le dispositif de recherche français au service de l'environnement et du développement apparaît comme potentiellement bien conçu et riche en compétences. II s'agit de faire jouer habilement et intelligemment les acteurs (chercheurs de divers instituts : Orstom, Cirad, CNRS, etc.), par exemple à travers une politique et une programmation scientifiques bien conçues. Ainsi, l'effort de programmation scientifique de l'Orstom nous semble relever d'une telle démarche. Une part de ce qui est présenté dans ce texte a d'ailleurs trouvé son inspiration dans ce travail de programmation (sans oublier, évidemment de nombreuses lectures non mentionnées ici, par exemple la lecture assidue de Natures, Sciences, Sociétés). L'expérience mériterait d'être étendue pour élaborer une véritable politique scientifique interorganisme qui identifierait mieux la contribution française à ces recherches et rendrait l'ensemble du dispositif plus cohérent et pertinent ; tout en sachant bien qu'au regard de ceux d'autres pays du Nord ce dispositif est déjà très original et sans doute mieux adapté à l'idée de coopération scientifique (par exemple, la pratique de la * recherche safari - n'est pas très fréquente dans la communauté scientifique française, cette position est largement la conséquence d'une idée de la recherche en coopération héritée de notre histoire, de l'existence d'instituts spécialisés et compétents comme l'Orstom et le Cirad). En tout état de cause, le travail récent du Comité national de coordination pour la recherche au service du développement et celui de ses groupes de réflexion constitue une contribution importante (Rapport 1996). II serait intéressant qu'en matière de recherche sur l'environnement un effort du même ordre soit fait et qu'il y ait ensuite confrontation et ajustement de l'ensemble de ces réflexions pour faire émerger un point de vue original sur la mise en relation explicite entre recherches sur (et pour) l'environnement et recherches au service du développement, ainsi que sur sa traduction en termes opérationnels. 


\section{Conclusion}

\section{Réalisme et nécessité de ces recherches dans le cadre d'une réelle compétence scientifique et d'une demande sociale existante, pressante, mais souvent diffuse, mal exprimée et le plus souvent non traduites en questions scientifiques}

La contribution de la recherche aux problèmes d'environnement et de développement est nécessaire, pertinente, de qualité et correspond, contrairement à des idées largement répandues, à une forte demande sociale. La lecture de cette demande n'est pas toujours commode, mais elle émerge, par exemple, à travers l'analyse de certaines enquêtes. Pour ce qui est de l'environnement, la demande est explicite : dans un récent sondage ${ }^{22}$ la demande de recherche scientifique sur ce thème arrive en deuxième position, et de près, après la santé (voir Santé et environnement). Pour ce qui est du développement, plus spécifiquement du développement durable et de l'attention à prêter aux problèmes de pays en développement, la demande est moins clairement explicitée tout simplement parce qu'elle est plus compliquée, moins intuitive et surtout non formulée, par exemple dans les enquêtes d'opinion. De plus, comme pour le mot environnement la polysémie du mot développement ne facilite pas la compréhension.

Pour toutes ces raisons, il peut y avoir une mauvaise appréciation par la communauté scientifique et par les autorités politiques de l'intérêt primordial de nos sociétés pour que les chercheurs abordent

\section{Santé et environnement}

Actuellement, la priorité est explicitement donnée à la santé et à l'environnement. Cependant, la question du développement n'a pas été posée explicitement et souvent sous le terme environnement se cachent évidemment des préoccupations liées au développement. On retrouve également ces préoccupations sous d'autres expressions, en partie évidemment en santé (lutte contre les grandes maladies), mais aussi pour reprendre les expressions employées : recherches sur de nouvelles sources d'énergie, recherches appliquées à des produits de la vie quotidienne, rendre les déserts fertiles, vaincre la faim dans le monde, sont des préoccupations prioritaires apparaissant dans ce sondage.

les questions du développement et même quelquefois encore celles de l'environnement. De plus, les fortes relations et même la dualité que nous venons de montrer entre environnement et développement nécessite une approche conjointe des recherches. Sur le plan de la méthode, ignorer l'importance des recherches sur le développement risque d'affaiblir les recherches sur l'environnement et la portée de leurs résultats ; négliger l'approche scientifique de l'environnement peut nuire aux recherches au service du développement et, plus profondément, à leurs traductions en termes techniques, politiques ou réglementaires.

Pertinence scientifique, nécessité méthodologique et légitimité sociale devraient conduire à faire que ces domaines de recherche soient des secteurs d'importance à la fois pour la communauté scientifique et les autorités politiques.
22 - Ce que les Français attendent de la science sondage exclusif SofresEurêka (1995). Eurêka 5 18-20 